\title{
Image of the month: Gallstone ileus
}

\author{
Authors: Chia-Ming Chang, ${ }^{A}$ Chian-Ze Peng, ${ }^{B}$ Yen-Chia Chen ${ }^{C}$ and Chorng-Kuang How ${ }^{D}$
}
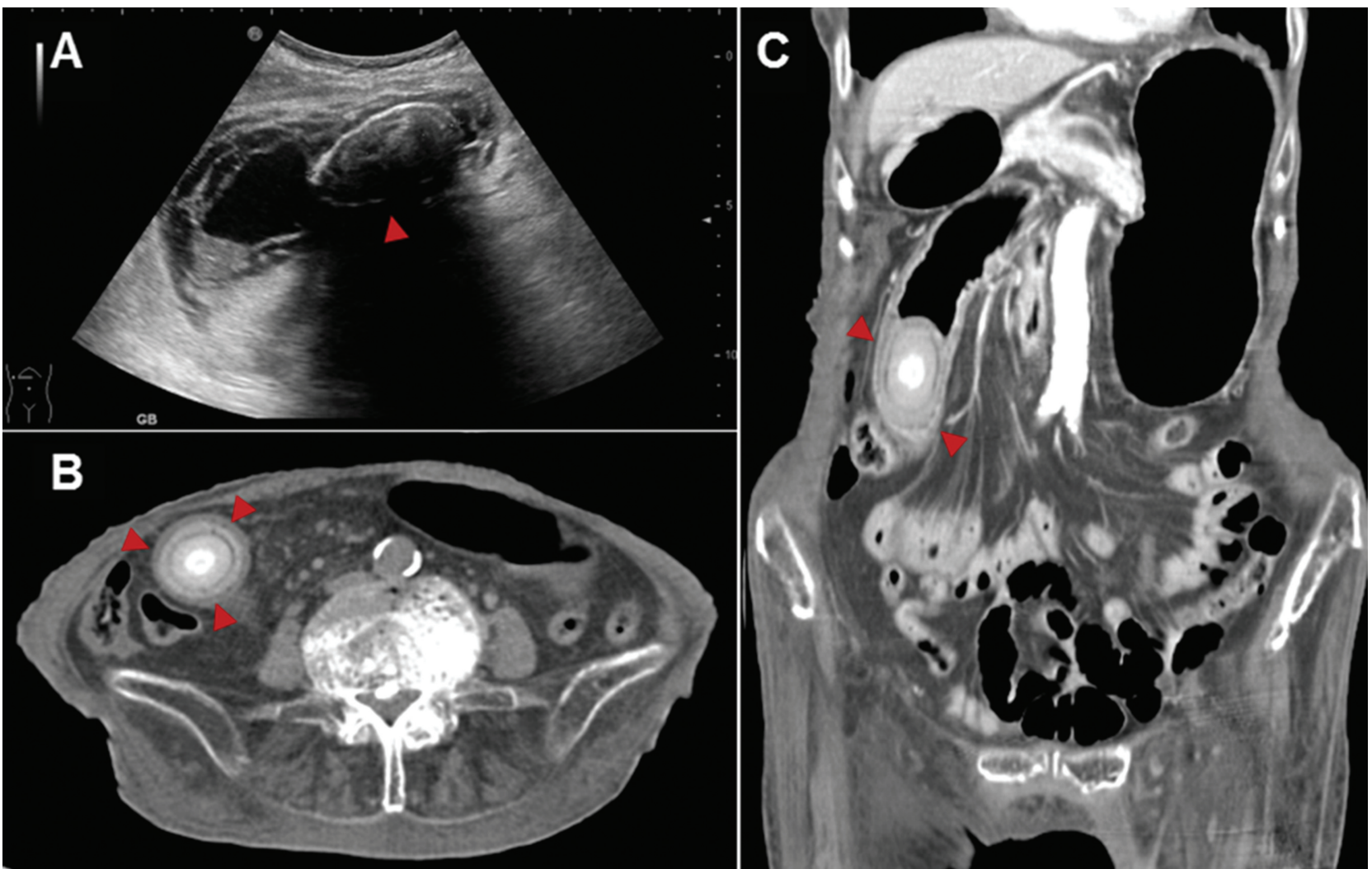

Fig 1. Gallstone image series. A - abdominal ultrasonography showing a huge gallstone within a thick-walled gallbladder (arrowhead); B and C - abdominal computerised tomography scan showing a calcified intra-luminal structure (arrowhead) in the proximal jejunum, which has caused proximal gas distension of the bowel, consistent with gallstone ileus.

A 97-year-old woman presented to our emergency department with nausea, vomiting and abdominal pain for about 2 days.

Authors: Aclinical lecturer, Taipei Veterans General Hospital, Taipei, Taiwan and National Yang-Ming University School of Medicine, Taipei, Taiwan; ${ }^{\text {B }}$ clinical lecturer, Taipei Veterans General Hospital, Taipei, Taiwan, Yuanshan \& Su-Ao Branch, Yilan, Taiwan and National Yang-Ming University School of Medicine, Taipei, Taiwan; ${ }^{C}$ assistant professor, Taipei Veterans General Hospital, Taipei, Taiwan and National Yang-Ming University School of Medicine, Taipei, Taiwan; ' associate professor, Taipei Veterans General Hospital, Taipei, Taiwan and National Yang-Ming University School of Medicine, Taipei, Taiwan
She had a history of ultrasound-guided percutaneous cholecystostomy following chronic cholecystitis with a huge gallstone 1 year earlier (Fig 1). Her physical examination showed hypoactive bowel sound and tympanic abdominal percussion. The abdominal radiograph showed no specific findings, except a distended stomach and dilated proximal small bowel loop. A follow-up abdominal computerised tomography $(\mathrm{CT})$ scan demonstrated a gallstone measuring about $4.6 \times 3.3 \mathrm{~cm}$ in the proximal jejunum, which caused proximal gas distension of the bowel, consistent with gallstone ileus (Fig 1). The patient underwent urgent enterolithotomy and she had an uneventful recovery.

Gallstone ileus is a rare bowel obstruction caused by cholelithiasis, which occurs more frequently in older women 
than men. The main cause of the development of a large gallstone in the intestine is a biliary enteric fistula. More than $70 \%$ of cases have cholecystoduodenal fistulas, followed by cholecystocolonic and cholecystogastric fistulas. Most gallstones affect the terminal ileum, which is the narrowest segment of the intestine. ${ }^{1}$

A preoperative diagnosis of gallstone ileus is challenging. Its nonspecific clinical presentation tends to delay diagnosis and treatment. The Rigler triad is the classic radiologic sign of gallstone ileus and consists of pneumobilia, intestinal obstruction and an ectopic gallstone. However, most gallstones are radiolucent and all three elements of the Rigler triad are present on plain radiographs in only $15 \%$ of cases. The Rigler triad is seen in up to $80 \%$ of cases when CT is used. ${ }^{2}$ Ultrasound is purported to have greater than $95 \%$ specificity and sensitivity in detecting gallstones within the gallbladder, but it is of limited use in cases of gallstone ileus because of gaseous distension of the bowel. ${ }^{3}$

Surgical intervention is the mainstay treatment strategy of gallstone ileus. The two-stage procedure (enterolithotomy followed by cholecystectomy and repair of the biliodigestive fistula after 4-6 weeks) is an alternative treatment suggested for patients with recurrent biliary symptoms. ${ }^{4}$ Nevertheless, a nonsurgical strategy, such as extracorporeal or electrohydraulic lithotripsy and the endoscopic removal of gallstones, has been reported..$^{5}$

\section{Conflict of interest}

The authors have no conflicts of interest to declare.

\section{Acknowledgements}

Written consent was obtained from the patient to publish the clinical details and images in this article.

\section{References}

1 Halabi WJ, Kang CY, Ketana N et al. Surgery for gallstone ileus: a nationwide comparison of trends and outcomes. Ann Surg 2014;259:329-35.

2 Lassandro F, Romano S, Ragozzino A et al. Role of helical CT in diagnosis of gallstone ileus and related conditions. AJR Am J Roentgenol 2005;185:1159-65.

3 Portincasa P, Moschetta A, Petruzzelli M et al. Symptoms and diagnosis of gallbladder stones. Best Pract Res Clin Gastronenterol 2006;20:1017-29.

4 Nuño-Guzmán CM, Marín-Contreras ME, Figueroa-Sánchez M, Corona JL. Gallstone ileus, clinical presentation, diagnostic and treatment approach. World J Gastronintest Surg 2016;8:65-76.

5 Pezzoli A, Maimone A, Fusetti N, Pizzo E. Gallstone ileus treated with non-surgical conservative methods: a case report. J Med Case Rep 2015;9:15

Address for correspondence: Dr Chian-Ze Peng, Emergency Department, Taipei Veterans General Hospital, No 201, Sec 2, ShiPai Rd, Beitou District, Taipei City, Taiwan 11217.

Email: cyberhunter700403@yahoo.com.tw 